\title{
AVALIAÇÃO DO DESEMPENHO DOS MODELOS DE PREDIÇÃO DA EROSÃO HÍDRICA USLE, RUSLE E WEPP PARA DIFERENTES CONDIÇÕES EDAFOCLIMÁTICAS DO BRASIL
}

\section{RICARDO S. S. AMORIM1 ${ }^{1}$, DEMETRIUS D. DA SILVA ${ }^{2}$, FERNANDO F. PRUSKI ${ }^{3}$, ANTÔNIO T. DE MATOS ${ }^{4}$}

\begin{abstract}
RESUMO: Este trabalho teve o objetivo de avaliar o desempenho dos modelos de predição das perdas de solo USLE, RUSLE e WEPP para diferentes condições edafoclimáticas brasileiras. Para alcançar o objetivo proposto, perdas de solo estimadas pelos referidos modelos em nove localidades do Brasil foram comparadas às perdas medidas em parcelas experimentais, por meio do coeficiente de correlação, da raiz do erro quadrático médio (RMSE), do índice de concordância de Willmott, do índice de confiança, do coeficiente de Nash-Sutcliffe e da eficiência dos modelos. As parcelas experimentais adotadas nos estudos de perdas de solo utilizados no presente trabalho apresentavam dimensões (de 38 a $1.875 \mathrm{~m}^{2}$ ) e tempo de coleta de dados (de 1 a 7 anos) variados. Observou-se diferença significativa entre a média geral de perdas de solo estimada pelos modelos e a medida no campo. O WEPP apresentou estimativas mais precisas em $46 \%$ das condições simuladas, seguido pelo RUSLE com $42 \%$ e USLE com $12 \%$, sendo que os indicadores estatísticos estudados mostraram que o modelo WEPP apresenta melhor desempenho quando comparado com os demais modelos. Apesar de a diferença entre os dados estimados e observados ocorrer para os três modelos, o WEPP, por apresentar melhor desempenho, coeficiente angular mais próximo da unidade na relação entre perdas de solo medidas no campo e estimadas pelo modelo e por ser embasado em processos físicos, demonstra ter maior potencial para fazer previsão da erosão para diferentes condições edafoclimáticas brasileiras.
\end{abstract}

PALAVRAS-CHAVE: erosão, conservação do solo, modelagem hidrológica.

\section{EVALUATION OF THE PERFORMANCE OF THE USLE, RUSLE AND WEPP SOILEROSION MODELS FOR DIFFERENT EDAPHOCLIMATIC CONDITIONS IN BRAZIL}

\begin{abstract}
The objective of this study was to evaluate the performance of USLE, RUSLE, and WEPP soil loss prediction models for different Brazilian soil and climatic conditions. To achieve this objective, soil losses estimated by the mentioned models in nine locations in Brazil were compared with the losses measured in the field by the following statistical parameters: correlation coefficient, root mean square error, Wilmott concordance index, confidence index, Nash-Sutcliffe coefficient, and efficiency coefficient models. The experimental plots adopted in studies of soil losses used in this study had varied dimensions $\left(38\right.$ to $\left.1875 \mathrm{~m}^{2}\right)$ and monitoring times and periods (01 to 07 years). There was a significant difference between the overall average of soil loss estimated by the models and the corresponding values measured in the field. Soil losses estimated by the WEPP model were more accurate in $46 \%$ of the cases, followed by RUSLE and USLE models with 42 and $12 \%$, respectively. The statistical indicators showed that the WEPP model had better performance when compared with the other models. Despite the difference between the observed and the measured data for the three models, the WEPP model, which presented highest performance, angular coefficient closer to the unity in the relation between soil losses measured in the field and estimated by the model, and because it is based on physical processes, demonstrates to have higher potential to predict erosion for different Brazilian edaphoclimatic conditions.
\end{abstract}

KEYWORDS: erosion, soil conservation, hydrological modeling.

\footnotetext{
${ }^{1}$ Prof. Adjunto, DSER/UFMT, Av. Fernando Correa da Costa, s/n, Cuiabá - MT, Fone (0XX65) 3615.8616, rsamorim@ufmt.br.

${ }^{2}$ Prof. Associado II, D.Sc., Departamento de Engenharia Agrícola, UFV, Viçosa - MG, david@ mail.ufv.br. Bolsista CNPq.

${ }^{3}$ Prof. Titular, Departamento de Engenharia Agrícola, UFV, Viçosa - MG, ffpruski@ mail.ufv.br. Bolsista CNPq.

${ }^{4}$ Prof. Associado, Departamento de Engenharia Agrícola, UFV, Viçosa - MG, atmatos@ mail.ufv.br. Bolsista CNPq. 


\section{INTRODUÇÃO}

A erosão do solo tem sido assunto de grande preocupação no Brasil e no mundo, em razão da rapidez com que se processa e por acarretar grandes prejuízos para diversas atividades econômicas e ao próprio meio ambiente. A erosão acelerada do solo origina-se da combinação da intensificação agrícola com eventos de chuvas intensas, a qual é responsável por aproximadamente $85 \%$ da degradação dos solos. Cerca de 1,5 bilhão de hectares, ou seja, aproximadamente $10 \%$ da superfície terrestre, já foram degradados pelo processo de erosão, o qual é geralmente ativado e acelerado pelo uso e manejo inadequados do solo (ANGIMA et al., 2003 citado por LEITE, 2007).

Existem vários métodos de pesquisas para se estudar e avaliar as ocorrências e consequências do processo de erosão hídrica dos solos. Dentre as metodologias disponíveis para avaliar a erosão do solo, os modelos de predição são fundamentais, pois uma vez comprovada a sua adequação e confiabilidade, são capazes de avaliar diferentes cenários de manejo do solo sem necessidade de testes de campo, normalmente custosos e demorados (AKSOY \& KAVVAS, 2005).

Segundo AMORIM (2003), diversos modelos matemáticos vêm sendo desenvolvidos e aperfeiçoados, desde a década de 50, com o intuito de prever a magnitude das perdas de solo por erosão, visando a implementar ferramentas que possibilitam avaliar as perdas de solo com propósitos de auxiliar no planejamento agrícola, principalmente em locais onde as perdas de solo são superiores aos limites toleráveis. Os modelos de predição de erosão do solo evoluíram de modelos empíricos, tais como a Universal Soil Loss Equation (USLE) e a Revised Universal Soil Loss Equation (RUSLE), para modelos baseados em princípios teóricos, como o Water Erosion Prediction Project (WEPP).

Vários trabalhos têm sido conduzidos para avaliar o desempenho dos modelos de predição de erosão, bem como validar esses modelos para diferentes condições de manejo e uso do solo. RISSE et al. (1993) aplicaram o modelo USLE em parcelas experimentais, observando erro médio de aproximadamente $60 \%$ em relação aos dados medidos de perdas de solo. ZANG et al. (1996) aplicaram o modelo WEPP para 34 cenários diferentes, em oito localidades nos Estados Unidos, e verificaram um erro médio de aproximadamente $61 \%$ nas perdas de solo. Para ambos os trabalhos, o erro relativo foi maior para elevados valores de perdas de solo.

TIWARI et al. (2000), trabalhando com os modelos WEPP, USLE e RUSLE, verificaram que o modelo WEPP apresentou erro médio de $2,01 \mathrm{~kg} \mathrm{~m}^{-2}$ e de $2,73 \mathrm{~kg} \mathrm{~m}^{-2}$ nas estimativas das perdas de solo quando se considera a média anual dos dados experimentais e os dados anuais isoladamente, respectivamente. Em geral, as eficiências dos modelos foram baixas em locais com baixos valores de perdas de solo, enquanto, em locais com elevadas perdas de solo, ocorreram melhores predições, sendo que os desempenhos dos modelos USLE e WEPP foram mais satisfatórios. As estimativas das perdas de solo médias anuais obtidas utilizando-se do WEPP foram melhores em $40 \%$ dos locais estudados quando comparadas com as estimativas obtidas com a USLE e RUSLE.

PIERI et al. (2007) avaliaram o desempenho do modelo WEPP na Itália para diferentes sistemas de uso e manejo do solo, encontrando eficiência aceitável quando não foi realizado ajuste dos parâmetros de entrada do modelo, com coeficientes de Nash-Sutcliffe (NS) iguais a 0,58 e 0,34 para perdas de água e solo, respectivamente. No entanto, quando se fez o ajuste da condutividade hidráulica efetiva e da tensão crítica de cisalhamento, as estimativas melhoraram expressivamente, apresentando valores de NS iguais a 0,82 e 0,88 para perdas de água e solo, respectivamente.

Tendo em vista que os modelos de predição de erosão existentes foram desenvolvidos e ajustados para condições de clima temperado, condições estas bem diferentes do clima tropical, torna-se de fundamental importância a verificação da aplicabilidade destes modelos para as condições edafoclimáticas brasileiras, antes de serem extensivamente utilizados para a predição da erosão. No Brasil, poucos estudos têm sido realizados objetivando a avaliação e a validação de modelos de perdas de solo, principalmente WEPP e RUSLE. 
Na literatura, foram encontrados alguns trabalhos de aplicação dos modelos; dentre os mais recentes, podem-se citar os trabalhos de DE JONG VAN LIER et al. (2005), AVANZI et al. (2008), SILVA et al. (2008), SERGIO et al. (2008) e CECÍLIO et al. (2009), realizados para algumas condições de manejo do solo, mas em sua maioria referiam-se apenas à aplicação dos modelos para estimativas das perdas de solo sem o objetivo de fazer comparação dos dados estimados com dados obtidos experimentalmente.

Em função do exposto, este trabalho teve como objetivo avaliar os modelos USLE, RUSLE e WEPP na predição das perdas de solo quando aplicados para diferentes condições edafoclimáticas brasileiras, sem ajuste nos parâmetros de entradas dos modelos.

\section{MATERIAL E MÉTODOS}

Para alcançar o objetivo do presente trabalho, valores de perdas de solo estimados pelos modelos USLE, RUSLE e WEPP foram comparados aos dados de perdas de solo obtidos em parcelas experimentais submetidas a chuvas naturais instaladas em Alagoinha - PB, Campinas - SP, Pindorama -SP, Dourados -MS, Goiânia -GO, Guaiba - RS, Planaltina - DF, Santa Maria - RS e em Viçosa -MG, em estudos realizados por SILVA et al. (1986), CASTRO et al. (1986), HERNANI et al. (1997), SILVA et al. (1997), ELTZ et al. (1997), DEDECEK et al. (1986) e SEGANFREDO et al. (1997), citados por AMORIM (2003).

Em todos os estudos de perdas de solo adotados no presente trabalho, utilizou-se a metodologia de fragmentação do volume de escoamento por meio de calha Geib, sendo a quantificação das perdas de solo feita pelo método direto preconizado por COGO (1978).

Os parâmetros necessários para a aplicação dos modelos foram levantados para cada um dos locais estudados. A escolha das nove localidades foi realizada com base na disponibilidade de registros de dados de perdas de solo em estudos prévios, as quais contemplaram uma grande heterogeneidade de condições climáticas, edáficas, comprimento e declividade das encostas e práticas de uso e manejo do solo, de forma a possibilitar a verificação do desempenho dos modelos para diversas condições edafoclimáticas existentes no Brasil.

Na Tabela 1, estão apresentadas as informações relativas aos locais utilizados para a realização do estudo com suas respectivas características quanto à classe de solo, manejo e uso do solo, dimensões, declividade das parcelas experimentais, bem como tempo, período e número de eventos durante o monitoramento. As informações relativas às características do solo na profundidade de 0 a $20 \mathrm{~cm}$ da superfície, para cada localidade, estão apresentadas na Tabela 2.

As estimativas das perdas de solo com base no modelo USLE foram obtidas pela aplicação da eq.(1):

$$
\mathrm{PS}=\mathrm{R} \text { K L S C P }
$$

em que,

PS - perda de solo média anual, $\mathrm{t} \mathrm{ha}^{-1} \mathrm{ano}^{-1}$;

$\mathrm{R}$ - fator de erosividade da chuva, $\mathrm{MJ} \mathrm{mm} \mathrm{ha}^{-1} \mathrm{ano}^{-1} \mathrm{~h}^{-1}$;

$\mathrm{K}$ - fator de erodibilidade do solo, $\mathrm{t} \mathrm{h} \mathrm{MJ}^{-1} \mathrm{~mm}^{-1}$;

$\mathrm{L}$ - fator de comprimento de rampa, adimensional;

$\mathrm{S}$ - fator de declividade de rampa, adimensional;

$\mathrm{C}$ - fator de uso e manejo do solo, adimensional, e

$\mathrm{P}$ - fator de práticas conservacionistas, adimensional. 
TABELA 1. Características de solo, manejo, declividade (S), dimensões (DP) e tempo e período de monitoramento (TPM), e número de eventos de chuvas (NE) das áreas experimentais nos locais utilizados para a realização do estudo. Characteristics of experimental plots used in this study: soil type, management, slope (S), dimensions (SD), monitoring times and periods (TPM) and rainfall events number (NE).

\begin{tabular}{|c|c|c|c|c|c|c|}
\hline Local & Solo & Manejo & $\mathrm{S}(\%)$ & $\mathrm{DP}\left(\mathrm{m}^{2}\right)$ & TPM & $\mathrm{NE}$ \\
\hline $\begin{array}{l}\text { Alagoinha } \\
\qquad(\mathrm{PB})^{1}\end{array}$ & $\begin{array}{l}\text { Nitossolo } \\
\text { Vermelho } \\
\text { eutroférrico }\end{array}$ & $\begin{array}{l}\text { Algodão morro abaixo (AMA) } \\
\text { Algodão em nível (AEN) } \\
\text { Milho em nível (MEN) } \\
\text { Cana-de-açúcar em sulco e em nível (CEN) } \\
\text { Solo descoberto (SD) }\end{array}$ & 12,0 & $\begin{array}{l}100,0 \\
(20 \times 5)\end{array}$ & $\begin{array}{c}3 \text { anos } \\
(1980-1983)\end{array}$ & NI \\
\hline $\begin{array}{l}\text { Campinas } \\
\qquad(\mathrm{SP})^{2}\end{array}$ & $\begin{array}{l}\text { Latossolo } \\
\text { Vermelho } \\
\text { distroférrico }\end{array}$ & $\begin{array}{l}\text { Milho plantado no sentido do declive para os } \\
\text { seguintes preparos do solo: } \\
\text { Preparo convencional - arado de disco e duas } \\
\text { gradagens niveladoras (MC-PC); } \\
\text { Preparo reduzido1 - duas gradagens niveladoras } \\
\text { (MC-PR1); } \\
\text { Preparo reduzido } 2 \text { - escarificador de cinco dentes } \\
\text { e duas gradagens niveladoras (MC-PR2); e } \\
\text { Preparo reduzido } 3 \text { - escarificador de três dentes e } \\
\text { duas gradagens niveladoras (MC-PR3). }\end{array}$ & 6,5 & $\begin{array}{l}1875,0 \\
(25 \times 75)\end{array}$ & $\begin{array}{c}3 \text { anos } \\
(1979-1982)\end{array}$ & NI \\
\hline $\begin{array}{l}\text { Dourados } \\
(\mathrm{MS})^{3}\end{array}$ & $\begin{array}{l}\text { Latossolo } \\
\text { Vermelho } \\
\text { eutroférrico }\end{array}$ & $\begin{array}{l}\text { Plantio de soja e trigo cultivados em sucessão com: } \\
\text { Escarificação + gradagem niveladora (ST-ES); } \\
\text { Gradagem pesada + niveladora (ST-GP); } \\
\text { Plantio direto (ST-PD), e } \\
\text { Sistema convencional, sem cobertura vegetal } \\
\text { (ST-SD). }\end{array}$ & 3,0 & $\begin{array}{c}77,0 \\
(22 \times 3,5)\end{array}$ & $\begin{array}{c}7 \text { anos } \\
(1987-1994)\end{array}$ & NI \\
\hline $\begin{array}{l}\text { Goiânia } \\
(\mathrm{GO})^{4}\end{array}$ & $\begin{array}{l}\text { Latossolo } \\
\text { Vermelho } \\
\text { distrófico }\end{array}$ & Solo descoberto (SD). & 4,0 & $\begin{array}{c}77,0 \\
(22 \times 3,5)\end{array}$ & 5 anos & 415 \\
\hline $\begin{array}{l}\text { Guaíba } \\
(\mathrm{RS})^{5}\end{array}$ & $\begin{array}{l}\text { Argissolo } \\
\text { Bruno- } \\
\text { Acinzentado } \\
\text { Alítico }\end{array}$ & $\begin{array}{l}\text { Sucessão de culturas de trigo em cultivo } \\
\text { convencional e de soja em cultivo mínimo (TCSM); } \\
\text { Solo descoberto em cultivo convencional (SD), e } \\
\text { Sucessão de culturas de trigo e soja em } \\
\text { cultivo convencional (TCSC). }\end{array}$ & 12,0 & $\begin{array}{c}66,0 \\
(22 \times 3)\end{array}$ & $\begin{array}{c}1 \text { ano } \\
(1975-1976)\end{array}$ & 132 \\
\hline $\begin{array}{l}\text { Pindorama } \\
(\mathrm{SP})^{2}\end{array}$ & $\begin{array}{l}\text { Argissolo } \\
\text { Vermelho- } \\
\text {-Amarelo }\end{array}$ & $\begin{array}{l}\text { Os mesmos manejos adotados nas parcelas } \\
\text { instaladas em Campinas acrescentado do preparo } \\
\text { com grade pesada e duas gradagens niveladoras } \\
\text { (MC-GP). }\end{array}$ & 10,8 & $\begin{array}{c}640,0 \\
(16 \times 40)\end{array}$ & $\begin{array}{c}5 \text { anos } \\
(1980-1985)\end{array}$ & NI \\
\hline $\begin{array}{l}\text { Planaltina } \\
\quad(\mathrm{DF})^{6}\end{array}$ & $\begin{array}{l}\text { Latossolo } \\
\text { Vermelho } \\
\text { distrófico }\end{array}$ & $\begin{array}{l}\text { Solo descoberto (SD) } \\
\text { Milho convencional (MC) } \\
\text { Soja convencional (SC) } \\
\text { Soja sem palha (SSP) } \\
\text { Soja plantio direto (SPD) }\end{array}$ & 5,5 & $\begin{array}{c}77,0 \\
(22 \times 3,5)\end{array}$ & $\begin{array}{c}6 \text { anos } \\
(1979-1985)\end{array}$ & NI \\
\hline $\begin{array}{l}\text { Santa Maria } \\
\quad(\mathrm{RS})^{7}\end{array}$ & $\begin{array}{l}\text { Argissolo } \\
\text { Vermelho- } \\
\text {-Amarelo }\end{array}$ & $\begin{array}{l}\text { Solo descoberto (SD) } \\
\text { Pousio invernal e milho no verão (PIMV) }\end{array}$ & 5,5 & $\begin{array}{c}77,0 \\
(22 \times 3,5)\end{array}$ & $\begin{array}{c}2 \text { anos } \\
(1993-1995)\end{array}$ & NI \\
\hline $\begin{array}{l}\text { Viçosa } \\
(\mathrm{MG})^{8}\end{array}$ & $\begin{array}{l}\text { Cambissolo } \\
\text { Háplico } \mathrm{Tb} \\
\text { distrófico }\end{array}$ & $\begin{array}{l}\text { Solo descoberto com preparo convencional no } \\
\text { sentido do declive e mantido descoberto (SDMA) } \\
\text { Solo com preparo convencional no sentido } \\
\text { transversal ao declive e mantido descoberto (SDEN) } \\
\text { Solo com preparo convencional e plantio de milho } \\
\text { no sentido do declive (MCMA) } \\
\begin{array}{l}\text { Solo com preparo convencional e plantio de milho } \\
\text { no sentido transversal ao declive (MCEN) }\end{array}\end{array}$ & 16,3 & $\begin{array}{c}38,5 \\
(11 \times 3,5)\end{array}$ & $\begin{array}{c}5 \text { anos } \\
(2002-2007)\end{array}$ & 227 \\
\hline
\end{tabular}

${ }^{1}$ SILVA et al., 1986; ${ }^{2}$ CASTRO et al., 1986; ${ }^{3}$ HERNANI et al., 1997; ${ }^{4}$ SILVA et al., 1997; ${ }^{5}$ ELTZ et al., 1977; ${ }^{6}$ DEDECEK et al., 1986; ${ }^{7}$ SEGANFREDO et al., $1997 ;{ }^{8}$ AMORIM (2003); ${ }^{9}$ NI - não foi informado o NE no trabalho. 
TABELA 2. Propriedades da camada superficial do solo $(0-20 \mathrm{~cm})$ para cada local estudado. Soil properties of top $200 \mathrm{~mm}$ soil layer at each location.

\begin{tabular}{|c|c|c|c|c|c|c|c|c|}
\hline \multirow{2}{*}{ Localidade } & \multirow{2}{*}{ Classe textural } & A.G. ${ }^{1}$ & A.F. $^{2}$ & Arg. $^{3}$ & Silte & M.O. $^{4}$ & Rocha & CTC $^{5}$ \\
\hline & & \multicolumn{6}{|c|}{ dag kg ${ }^{-1}$} & $\mathrm{cmol}_{\mathrm{c}} \mathrm{kg}^{-1}$ \\
\hline Alagoinha-PB & Franco Argilo Arenosa & 23 & 24 & 36 & 17 & 1,83 & 2 & 9,7 \\
\hline Campinas-SP & Argila pesada & 10 & 20 & 62 & 8 & 2,24 & 0 & 8,9 \\
\hline Dourados-MS & Argila pesada & 6 & 5 & 74 & 14 & 4,60 & 0 & 17,25 \\
\hline Goiânia-GO & Argila pesada & 7 & 5 & 72 & 16 & 3,78 & 0 & 10,5 \\
\hline Guaiba-RS & Franco Argilo Arenosa & 35 & 12 & 31 & 22 & 2,00 & 12 & 8,5 \\
\hline Pindorama-SP & Areia Franca & 25 & 64 & 9 & 2 & 1,55 & 0 & 4,1 \\
\hline $\begin{array}{l}\text { Planaltina-DF } \\
\text { DF }\end{array}$ & Argila pesada & 8 & 7 & 70 & 16 & 6,09 & $\overline{1}$ & 14,25 \\
\hline Santa Maria-RS & Franco arenoso & 43 & 30 & 15 & 12 & 1,19 & 0 & $\overline{5,4}$ \\
\hline Viçosa-MG & Franco arenoso & 29 & 30 & 18 & 23 & 2,44 & 0 & 4,13 \\
\hline
\end{tabular}

O fator de erosividade das chuvas foi obtido de levantamento de dados já publicados, uma vez que, para todas as localidades estudadas, este fator já havia sido determinado (Tabela 3) pelo método de análise de pluviogramas, segundo metodologia preconizada por WISCHMEIER \& SMITH (1978).

O fator erodibilidade do solo (K) para cada local foi determinado pela eq.(2), usada na construção do nomograma de WISCHMEIER \& SMITH (1978):

$$
\mathrm{K}=\frac{\left[2,110^{4}(12-\mathrm{OM}) \mathrm{M}^{1,14}+3,25(\mathrm{~s}-2)+2,5(\mathrm{p}-3)\right]}{100} 0,1318
$$

em que,

OM - conteúdo de matéria orgânica, dag $\mathrm{kg}^{-1}$;

$\mathrm{M}$ - parâmetro que representa a textura do solo (eq.(3));

$\mathrm{s}$ - classe de estrutura do solo, adimensional, e

$\mathrm{p}$ - permeabilidade do perfil, adimensional.

$$
\mathrm{M}=(\% \text { silte }+\% \text { areia fina })(100-\% \text { argila })
$$

Os valores referentes à classe de estrutura (s) e de permeabilidade (p) foram determinados segundo a classificação proposta por WISCHMEIER \& SMITH (1978), sendo que os valores de s foram obtidos com base nas informações de estrutura do solo contidas nos trabalhos de levantamento de solo para cada localidade, e os valores de p, com base na classificação da permeabilidade do solo em função da classe textural do solo.

O fator topográfico (LS) foi determinado utilizando-se da eq.(4):

$$
\mathrm{LS}=\left(\frac{\mathrm{L}}{22,13}\right)^{\mathrm{ms}}\left(65,41 \operatorname{sen}^{2} \alpha+4,56 \operatorname{sen} \alpha+0,065\right)
$$

em que,

L - comprimento da encosta, $\mathrm{m}$;

$\alpha$ - ângulo do declive, graus, e

ms - coeficiente de ajuste dependente da declividade do terreno (S); sendo 0,5 para $\mathrm{S}>5 \%$; 0,4 para $3,5 \leq \mathrm{S} \leq 5 \% ; 0,3$ para $1,0 \leq \mathrm{S} \leq 3,5 \%$; e 0,2 para áreas planas.

Os fatores de uso e manejo do solo $(\mathrm{C})$ e de práticas conservacionistas $(\mathrm{P})$ para as diferentes condições de uso e manejo do solo foram baseados em dados apresentados na literatura (WISCHMEIER \& SMITH, 1978; RENARD et al., 1997; dentre outras).

As perdas de solo estimadas utilizando o modelo RUSLE foram obtidas por meio do programa RUSLE 1.06 (RENARD et al., 1997). Para executar este programa, foi necessário definir 
três arquivos de bancos de dados CITY DATABASE, CROP DATABASE e OPERATIONS $D A T A B A S E$, os quais foram adaptados às condições edafoclimáticas e de uso e manejo do solo de cada local estudado, a partir das informações de solo, clima, manejo e uso do solo em cada local específico, sendo que os bancos de dados CROP DATABASE e OPERATIONS DATABASE foram montados com base nas informações disponíveis nos locais de estudo e no banco de dados da RUSLE.

Os parâmetros de solo utilizados para a determinação do fator de erodibilidade do solo foram os apresentados na Tabela 2. Para a determinação do fator $\mathrm{C}$, as informações referentes às datas, às sequências e aos tipos de cultivos realizados nas parcelas experimentais foram obtidas na descrição apresentada nos trabalhos de perdas de solo utilizados na avaliação dos modelos. Para a aplicação do WEPP, foi necessária a elaboração de arquivos de clima, de solo, de manejo e uso do solo e topográfico, os quais foram baseados em registros de dados locais. Todos esses arquivos foram colocados no formato específico do software WEPP for Windows versão 95/98/NT. As informações necessárias para a construção do arquivo de solo utilizado no WEPP, tais como conteúdo de areia, silte, argila, rocha, matéria orgânica, classe textural e capacidade de troca catiônica do perfil de solo, foram obtidas de trabalhos de levantamento de solos para cada local estudado.

Os arquivos de uso e manejo do solo com as informações referentes ao crescimento de plantas, condições iniciais, decomposição de resíduos e práticas de manejo do solo foram montados com base nas informações disponíveis em cada local e na base de dados disponível no WEPP. As informações referentes às datas, às sequências e aos tipos de cultivos realizados nas parcelas experimentais foram obtidas na descrição apresentada nos trabalhos de perdas de solo. A base de dados de cultivo e manejo do solo, inclusa no WEPP, foi selecionada de forma a melhor representar as práticas de cultivo e manejo adotadas nas parcelas experimentais de cada local estudado.

$\mathrm{O}$ arquivo de dados climáticos diários para a entrada no modelo WEPP foi gerado com auxílio do programa CLIGEN 4.3. A média e o desvio-padrão da precipitação, coeficiente de assimetria da precipitação total diária, probabilidades mensais de ocorrência de dias com chuva após dias com chuva e de ocorrência de dias sem chuva após dias com chuva, média e desvio-padrão das temperaturas máxima e mínima mensal, média e desvio-padrão da radiação solar mensal e média mensal da temperatura do ponto de orvalho foram obtidos a partir de dados climáticos coletados em estações climatológicas existentes em cada localidade em estudo.

As probabilidades de ocorrência de dias com chuva seguidos de dia com chuva e de dias com chuva seguidos de dia sem chuva para cada mês foram estimadas a partir do número de dias chuvosos e sem chuva do mês, do número de dias chuvosos após dias sem chuva e do número de dias chuvosos após dia com chuva. Para a estimativa da radiação solar, utilizou-se da metodologia recomendada pela FAO 56. A temperatura no ponto de orvalho foi calculada utilizando os dados de temperatura e umidade relativa.

A avaliação da eficiência dos modelos foi realizada por meio dos seguintes parâmetros estatísticos: coeficiente de correlação, raiz do quadrado médio do erro (eq.(5)), índice de concordância de Wilmott (eq.(6)), índice de confiança, coeficiente de Nash-Sutcliffe (eq.(7)) e coeficiente de eficiência dos modelos.

$$
\operatorname{RMSE}=\sqrt{\frac{\sum_{\mathrm{i}=1}^{\mathrm{n}}\left(\mathrm{Y}_{\mathrm{obs} s_{i}}-\mathrm{Y}_{\mathrm{est} \mathrm{t}_{\mathrm{i}}}\right)^{2}}{\mathrm{n}}}
$$

em que,

RMSE - raiz do quadrado médio do erro;

$Y_{\text {obs }}$ - valor observado;

$\mathrm{Y}_{\text {est }}$ - valor estimado, e

$\mathrm{n}$ - número total de pares de valores observados e estimados. 


$$
\begin{aligned}
& \mathrm{d}=1-\frac{\sum_{\mathrm{i}=1}^{\mathrm{n}}\left(\mathrm{Y}_{\mathrm{est} \mathrm{t}_{\mathrm{j}}}-\mathrm{Y}_{\mathrm{obs} s_{\mathrm{i}}}\right)^{2}}{\sum_{\mathrm{i}=1}^{\mathrm{n}}\left(\left|\mathrm{Y}_{\mathrm{ast} \mathrm{t}_{\mathrm{i}}}-\overline{\mathrm{Y}}\right|+\left|\mathrm{Y}_{\mathrm{obs} s_{\mathrm{i}}}-\overline{\mathrm{Y}}\right|\right)^{2}} \\
& \mathrm{NS}=1-\frac{\sum_{\mathrm{i}=1}^{\mathrm{n}}\left(\mathrm{Y}_{\mathrm{obs}}-\mathrm{Y}_{\mathrm{est}}\right)^{2}}{\sum_{\mathrm{i}=1}^{\mathrm{n}}\left(\mathrm{Y}_{\mathrm{obs}}-\overline{\mathrm{Y}}\right)^{2}}
\end{aligned}
$$

em que,

d - índice de concordância;

$\overline{\mathrm{Y}}$ - média dos valores observados, e

NS - coeficiente de Nash-Sutcliffe.

O índice de confiança (c) foi obtido pelo produto entre o coeficiente de correlação de Pearson e o índice de concordância.

A análise da eficiência dos modelos, proposta por NEARING (2000), consistiu nos seguintes passos: a) determinar a diferença relativa entre os dados de perdas de solo medidos e estimados Rdiff $_{\mathrm{s}}$ (eq.(8)); b) determinar o intervalo de ocorrência para cada valor de perda de solo medido $\operatorname{Rdiff}_{\text {occ }}$ (eq.(9)); c) determinar o número de estimativas em que o valor de Rdiff fica dentro do

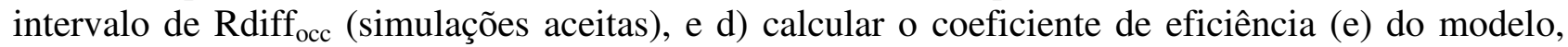
obtido a partir da relação entre o número de simulações aceitas e o número de simulações realizadas, que representa qual a fração das estimativas realizadas qual estão dentro do intervalo de ocorrência da perda de solo medida em função da sua variabilidade.

$$
\begin{aligned}
& \text { Rdiff }_{\mathrm{s}}=\frac{\mathrm{Y}_{\mathrm{est} \mathrm{t}_{\mathrm{i}}}-\mathrm{Y}_{\mathrm{obs} s_{\mathrm{i}}}}{\mathrm{Y}_{\mathrm{ast} \mathrm{t}_{\mathrm{i}}}+\mathrm{Y}_{\mathrm{ob} s_{\mathrm{i}}}} \\
& \text { Rdiff }_{\mathrm{occ}}=\mathrm{a} \log (\mathrm{a})+\mathrm{b}
\end{aligned}
$$

em que,

$\mathrm{a}$ e $\mathrm{b}$ - parâmetros de ajuste da equação apresentando os seguintes valores: $\mathrm{a}=+0,236 \mathrm{e} \mathrm{b}=$ $-0,641$ para o limite inferior do intervalo de $95 \%$; e $a=-0,179$ e $b=+0,416$ para o limite superior do intervalo de $95 \%$.

Além disso, realizou-se o teste t para fazer um estudo comparativo das médias de perdas de solo obtidas experimentalmente e estimadas pelos modelos (USLE, RUSLE e WEPP), a 5\% de probabilidade. Tendo em vista que os dados originais não apresentavam normalidade, fez-se a transformação dos dados para a obtenção da normalidade e possibilitar a aplicação do teste t.

\section{RESULTADOS E DISCUSSÃO}

$\mathrm{Na}$ Tabela 3, estão apresentados os valores das perdas de solo estimadas, utilizando-se dos modelos USLE, RUSLE e WEPP, e a magnitude do desvio das estimativas em relação aos dados observados para cada condição de uso e manejo do solo, adotada nas diferentes localidades em estudo. Pode-se observar que nenhum dos modelos utilizados se mostrou mais preciso para todas as condições de uso e manejo do solo analisadas, observando-se que as estimativas obtidas, utilizando-se do modelo WEPP, foram mais precisas em 45,5\% das condições simuladas, seguidas pelas obtidas pela RUSLE com $42,4 \%$ e USLE com 12,1\%. Com relação à magnitude do desvio, pode-se observar que, para a USLE, ocorreu variação de $-2,23$ a $25,04 \mathrm{~kg} \mathrm{~m}^{-2}$; para a RUSLE, entre $-0,53 \mathrm{e}$ $25,50 \mathrm{~kg} \mathrm{~m}^{-2}$, e para o WEPP, entre $-3,57$ e $6,66 \mathrm{~kg} \mathrm{~m}^{-2}$.

Fazendo-se uma análise de tendência para os diversos tratamentos adotados nas diferentes localidades, observaram-se que, de maneira geral, as estimativas obtidas pelos modelos apresentaram tendência semelhante à dos dados observados, com exceção dos resultados obtidos para Campinas e Pindorama, para as quais os modelos USLE e WEPP não conseguiram representar o efeito dos diferentes tipos de preparo de solo adotados para estas localidades (Tabela 3). 
TABELA 3. Valores anuais médios de perdas de solos (PS) observados no campo e estimados pelos modelos USLE, RUSLE e WEPP, e correspondentes magnitudes do erro em relação aos dados observados para as nove localidades e diferentes condições de uso e manejo solo. Mean annual values of soil loss (PS) observed in the field and estimated by the USLE, RUSLE and WEPP models and their corresponding error magnitudes in relation to the observed data for each site and different conditions of soil cropping and management.

\begin{tabular}{|c|c|c|c|c|c|c|c|c|c|}
\hline \multirow{3}{*}{ Localidades } & \multirow{3}{*}{ Solo } & \multirow{3}{*}{$\begin{array}{l}\text { Uso e Manejo } \\
\text { do Solo }\end{array}$} & \multirow{2}{*}{$\begin{array}{c}\text { Observada } \\
\text { OS } \\
\end{array}$} & \multicolumn{2}{|c|}{ USLE } & \multicolumn{2}{|c|}{ RUSLE } & \multicolumn{2}{|c|}{ WEPP } \\
\hline & & & & OS & ERRO & PS & ERRO & PS & ERRO \\
\hline & & & & & & $\mathrm{m}^{-2}$ & & & 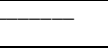 \\
\hline \multirow{5}{*}{ Alagoinha - PB } & \multirow{5}{*}{$\begin{array}{c}\text { Nitossolo Vermelho } \\
\text { eutroférrico }\end{array}$} & AMA & 3,19 & 8,05 & $+4,86$ & 6,05 & $+2,86$ & 1,11 & $-2,08$ \\
\hline & & AEN & 1,30 & 4,02 & $+2,72$ & 2,91 & $+1,61$ & 1,11 & $-0,19$ \\
\hline & & MEN & 1,09 & 2,00 & $+0,91$ & 1,35 & $+0,26$ & 1,09 & $+0,00$ \\
\hline & & CEN & 0,22 & 0,88 & $+0,66$ & - & - & 0,43 & $+0,21$ \\
\hline & & SD & 9,38 & 11,76 & $+2,38$ & 10,31 & $+0,93$ & 5,81 & $-3,57$ \\
\hline \multirow{4}{*}{ Campinas - SP } & \multirow{4}{*}{$\begin{array}{c}\text { Latossolo Vermelho } \\
\text { distroférrico }\end{array}$} & MC-PC & 0,30 & 5,93 & $+5,63$ & 4,04 & $+3,74$ & 4,46 & $+4,16$ \\
\hline & & MC-PR1 & 0,16 & 5,93 & $+5,77$ & 1,19 & $+1,03$ & 3,38 & $+3,22$ \\
\hline & & MC-PR2 & 0,20 & 5,93 & $+5,73$ & 1,73 & $+1,53$ & 4,12 & $+3,92$ \\
\hline & & MC-PR3 & 0,30 & 5,93 & $+5,63$ & 3,81 & $+3,51$ & 4,04 & $+3,74$ \\
\hline \multirow{4}{*}{ Dourados - MS } & \multirow{4}{*}{$\begin{array}{c}\text { Latossolo Vermelho } \\
\text { eutroférrico }\end{array}$} & ST-ES & 0,27 & 0,72 & $+0,45$ & 0,79 & $+0,52$ & 0,70 & $+0,43$ \\
\hline & & ST-GP & 0,59 & 0,72 & $+0,13$ & 1,70 & $+1,11$ & 0,81 & $+0,22$ \\
\hline & & ST-PD & 0,07 & 0,72 & $+0,65$ & 0,21 & $+0,14$ & 0,57 & $+0,50$ \\
\hline & & ST-SD & 0,78 & 1,34 & $+0,56$ & 4,04 & $+3,26$ & 1,77 & $+0,99$ \\
\hline Goiânia - GO & $\begin{array}{c}\text { Latossolo Vermelho } \\
\text { distrófico } \\
\end{array}$ & SD & 4,60 & 5,12 & $+0,52$ & 6,95 & $+2,35$ & 3,35 & $-1,25$ \\
\hline \multirow{3}{*}{ Guaíba - RS } & \multirow{3}{*}{$\begin{array}{l}\text { Argissolo Bruno- } \\
\text {-Acinzentado alítico }\end{array}$} & TCSM & 0,28 & 8,35 & $+8,07$ & 0,21 & $-0,07$ & 5,60 & $+5,32$ \\
\hline & & $\mathrm{SD}$ & 10,69 & 33,39 & $+22,70$ & 19,95 & $+9,26$ & 16,29 & $+5,60$ \\
\hline & & TCSC & 0,33 & 18,03 & $+17,70$ & 0,49 & $+0,16$ & 6,38 & $+6,05$ \\
\hline \multirow{5}{*}{ Pindorama - SP } & \multirow{5}{*}{$\begin{array}{c}\text { Argissolo Vermelho- } \\
\text {-Amarelo }\end{array}$} & MC-PC & 4,98 & 11,81 & $+6,83$ & 8,52 & $+3,54$ & 7,09 & $+2,11$ \\
\hline & & MC-GP & 5,61 & 11,81 & $+6,20$ & 10,76 & $+5,15$ & 7,06 & $+1,45$ \\
\hline & & MC-PR1 & 2,54 & 11,81 & $+9,27$ & 2,22 & $-0,32$ & 7,00 & $+4,46$ \\
\hline & & MC-PR2 & 2,23 & 11,81 & $+9,58$ & 1,70 & $-0,53$ & 7,04 & $+4,81$ \\
\hline & & MC-PR3 & 2,64 & 11,81 & $+9,17$ & 2,47 & $-0,17$ & 7,04 & $+4,40$ \\
\hline \multirow{5}{*}{ Planaltina - DF } & \multirow{5}{*}{$\begin{array}{c}\text { Latossolo Vermelho } \\
\text { distrófico }\end{array}$} & SD & 5,30 & 7,22 & $+1,92$ & 9,86 & $+4,56$ & 6,64 & $+1,34$ \\
\hline & & $\mathrm{MC}$ & 2,88 & 0,65 & $-2,23$ & 2,47 & $-0,41$ & 2,97 & $+0,09$ \\
\hline & & $\mathrm{SC}$ & 0,83 & 3,90 & $+3,07$ & 2,49 & $+1,66$ & 3,34 & $+2,51$ \\
\hline & & SSP & 0,62 & 3,90 & $+3,28$ & 1,84 & $+1,22$ & 4,01 & $+3,39$ \\
\hline & & SPD & 0,43 & 1,80 & $+1,37$ & 0,85 & $+0,42$ & 0,45 & $+0,02$ \\
\hline \multirow{2}{*}{ Santa Maria - RS } & \multirow{2}{*}{$\begin{array}{l}\text { Argissolo Vermelho- } \\
\text {-Amarelo }\end{array}$} & SD & 20,13 & 18,66 & $-1,47$ & 22,42 & $+2,29$ & 26,59 & $+6,46$ \\
\hline & & PIMV & 0,54 & 2,35 & $+1,81$ & 1,86 & $+1,32$ & 0,82 & $+0,28$ \\
\hline \multirow{4}{*}{ Viçosa - MG } & \multirow{4}{*}{$\begin{array}{c}\text { Cambissolo Háplico } \\
\text { Tb distrófico }\end{array}$} & SDMA & 3,61 & 15,28 & $+11,67$ & 29,15 & $+25,54$ & 10,28 & $+6,67$ \\
\hline & & SDEN & 5,74 & 30,78 & $+25,04$ & 14,57 & $+8,83$ & 10,27 & $+4,53$ \\
\hline & & MCMA & 0,27 & 11,59 & $+11,32$ & 7,40 & $+7,13$ & 3,79 & $+3,52$ \\
\hline & & MCEN & 0,05 & 7,58 & $+7,53$ & 3,59 & $+3,54$ & 5,74 & $+5,69$ \\
\hline
\end{tabular}

*valor em negrito indica o valor estimado mais próximo do valor observado experimentalmente. Não foi possível aplicar o modelo RUSLE para estimativas das perdas de solo para a condição de uso e manejo do solo CEN.

Para a USLE, isto ocorreu devido à inexistência de valores dos fatores C e P para todos os tipos de preparo do solo, sendo assumido valor constante para os diferentes tipos de preparo do solo adotados em Campinas e Pindorama. Já para o modelo WEPP, isto pode ter ocorrido devido à falta de informações na base de dados do WEPP referentes aos tipos de preparo e dos implementos utilizados em Campinas e Pindorama, uma vez que as condições de preparo e cobertura do solo 
afetam significativamente sua rugosidade e, por conseguinte, as perdas de solo (SILVA et al., 2005; CARVALHO FILHO et al., 2007; MARTINS FILHO et al., 2009; LEITE et al., 2009).

Na Tabela 4, pode-se verificar que todos os modelos de predição da erosão apresentaram média das estimativas de perdas de solo estatisticamente superior à média da perda de solo obtida experimentalmente, indicando uma tendência de superestimativa das perdas de solo. No entanto, houve diferença significativa entre as perdas estimadas pelo modelo USLE e a dos modelos RUSLE e WEPP; e entre os dois últimos modelos, as médias das estimativas foram estatisticamente iguais. Com relação aos desvios, pode-se observar que não houve diferença significativa entre os desvios médios obtidos pelos modelos RUSLE e WEPP, entretanto os desvios médios destes dois modelos foram significativamente menores em relação aos obtidos pelo modelo USLE.

Analisando os valores dos coeficientes de correlação, da RMSE, do coeficiente de eficiência de Nash-Sutcliffe e dos índices de concordância e confiança, observou-se que o modelo WEPP apresentou os melhores valores, seguido da RUSLE e, por último, da USLE. O valor do coeficiente de eficiência de Nash-Sutcliffe (NS) encontrado para o modelo WEPP foi um pouco inferior ao obtido por PIERI et al. (2007), que obtiveram coeficientes de Nash-Sutcliffe iguais a 0,34 para perdas de solo, podendo, desta forma, ser considerada uma eficiência aceitável, uma vez que não foi realizado nenhum processo de calibração dos parâmetros dos modelos.

Com relação ao índice de concordância (d) de Wilmott, que expressa a exatidão das estimativas em relação aos valores observados, verifica-se que o modelo apresentou maior nível de exatidão, apresentando o valor de 'd' mais próximo da unidade $(\mathrm{d}=0,86)$.

TABELA 4. Parâmetros estatísticos para a avaliação das estimativas das perdas de solo pelos modelos USLE, RUSLE e WEPP, considerando todas as condições edafoclimáticas estudadas. Statistical parameters for evaluation of soil losses estimates by USLE, RUSLE and WEPP models, considering all edaphoclimatics conditions studied.

\begin{tabular}{lcccc}
\hline \multirow{2}{*}{ Parâmetros estatísticos } & \multicolumn{3}{c}{ PS estimada } & \multirow{2}{*}{ PS experimental } \\
\cline { 2 - 4 } & USLE & RUSLE & WEPP & \\
\hline Média das PS $\left(\mathrm{kg} \mathrm{m}^{-2}\right)^{*}$ & $8,53 \mathrm{a}$ & $5,87 \mathrm{~b}$ & $5,19 \mathrm{~b}$ & \multirow{2}{*}{2,79} \\
\hline Média da magnitude do erro $\left(\mathrm{kg} \mathrm{m}^{-2}\right)^{*}$ & $5,97 \mathrm{a}$ & $3,09 \mathrm{~b}$ & $2,83 \mathrm{~b}$ \\
\hline Coeficiente de correlação $(\mathrm{r})$ & 0,60 & 0,73 & 0,86 \\
\hline RMSE & 8,53 & 5,53 & 3,54 \\
\hline Coeficientes de Nash-Sutcliffe $(\mathrm{NS})$ & $-3,40$ & $-0,86$ & 0,24 \\
\hline Indice de ajustamento $(\mathrm{d})$ & 0,56 & 0,73 & 0,86 \\
\hline Índice de confiança $(\mathrm{c})$ & 0,33 & 0,54 & 0,74 \\
\hline Eficiência dos modelos $\left(\mathrm{e}_{95}\right)$ & 0,27 & 0,50 & 0,49 \\
\hline * valores seguidos de mesma letra minúscula não diferem, pelo teste t, ao nível de $5 \%$ de probabilidade
\end{tabular}

Segundo a classificação apresentada em BIUDES et al. (2008), os índices de confiança obtidos para os modelos WEPP, RUSLE e USLE são: bom, sofrível e péssimo, respectivamente.

Já com relação ao parâmetro eficiência dos modelos ( $\mathrm{e}_{95}$ ), verificou-se que RUSLE e WEPP foram semelhantes e expressivamente superiores à USLE. Os valores de e95 obtidos no presente trabalho para os modelos WEPP e RUSLE podem ser considerados satisfatórios, tendo como base o estudo realizado por NEARING (2000) que, utilizando o mesmo critério de avaliação adotado neste estudo, observou valores de $e_{95}$ iguais a 0,56 e 0,66 para os modelos RUSLE e WEPP, respectivamente.

Desta forma, segundo os parâmetros de avaliação estatística dos modelos apresentados na Tabela 4, verifica-se que o modelo WEPP, de maneira geral, apresenta estimativas mais precisas quando comparadas às obtidas pelos outros dois modelos estudados, embora sejam estatisticamente diferentes dos valores obtidos experimentalmente. Esses resultados discordam das observações 
feitas em estudos realizados nos EUA, nos quais os modelos USLE e RUSLE apresentaram um erro médio absoluto menor do que o encontrado no presente trabalho, e, em alguns estudos, esses modelos apresentaram melhor desempenho do que o modelo WEPP nas estimativas das perdas de solo (RISSE et al., 1993; TIWARI et al., 2000). Esta discordância pode estar associada à ampla base de dados existente naquele país para a aplicação da USLE e RUSLE, bem como pelo fato de se tratar de modelos empíricos que foram desenvolvidos para as condições edafoclimáticas dos EUA. Esta hipótese corrobora a afirmativa de que os modelos empíricos, quando utilizados para as condições nas quais foram desenvolvidos, podem ser mais precisos do que os modelos com maiores níveis de complexidade.

Os resultados do presente trabalho também discordam dos obtidos por CECÍLIO et al. (2009), que verificaram melhor desempenho do modelo RUSLE em comparação ao WEPP, quando aplicados em um Latossolo Vermelho-Amarelo. Esta discordância se deve ao fato de as equações utilizadas pelo modelo WEEP, para estimar os parâmetros de solo, principalmente, condutividade hidráulica efetiva, erodibilidade no sulco e tensão crítica de cisalhamento, não serem adequadas para esta classe de solo por desconsiderar o efeito do seu caráter oxídico sobre as características físico-hídricas do solo. Esta hipótese pode ser confirmada fazendo uma análise comparativa das diferentes classes de solo englobadas no presente estudo (Tabela 3), na qual se verifica que a maioria dos piores desempenhos do modelo WEPP, comparativamente aos modelos RUSLE e/ou USLE, ocorreram para as parcelas que foram conduzidas em Latossolos.

Nas Figuras 1a, 1b e 1c, pode-se confirmar o indicativo de melhores estimativas das perdas de solo utilizando-se do modelo WEPP comparativamente aos modelos RUSLE e USLE, como observado na Tabela 4, uma vez que as menores magnitudes dos desvios entre os valores estimados e observados experimentalmente foram obtidas com uso deste modelo. Os maiores desvios observados nas estimativas obtidas pelos modelos USLE e RUSLE podem ser explicados, em parte, pelo caráter empírico destes modelos, os quais não consideram a distribuição temporal das chuvas ao longo do ano e, principalmente, no caso da USLE, devido à falta de base de dados para a determinação precisa dos fatores $\mathrm{C}$ e $\mathrm{P}$ para as condições brasileiras de uso e manejo do solo, nas quais os modelos foram aplicados.

Pode-se ainda observar, nas Figuras 1a, 1b e 1c, que a maioria dos pontos se localiza acima da linha de desvio nulo, evidenciando a tendência de superestimativa das perdas de solo pelos três modelos para as condições de uso e manejo do solo consideradas nas simulações.

Para os modelos USLE e RUSLE, esta superestimativa deve-se principalmente às incertezas na determinação dos fatores $\mathrm{K}, \mathrm{C}$ e P. Para o modelo WEPP, a superestimativa das perdas de solo deve-se à inadequação, para as condições estudadas, das equações utilizadas pelo modelo para estimar alguns parâmetros, principalmente aqueles relativos ao solo, tais como: erodibidade do solo, tensão crítica de cisalhamento e condutividade hidráulica do solo (AMORIM, 2003).

A inadequação das equações utilizadas para estimar os parâmetros relativos ao solo, tanto para o modelo WEPP quanto para a RUSLE e USLE, deve-se provavelmente à não consideração de características do solo, como, por exemplo, o caráter oxídico, que tem grande influência sobre a formação de microagregados, fazendo com que, mesmo em solos com elevados conteúdos de argila, se possa ter elevada permeabilidade (CANTALICE et al. 2005; BEZERRA \& CANTALICE, 2006; NUNES \& CASSOL, 2008). 

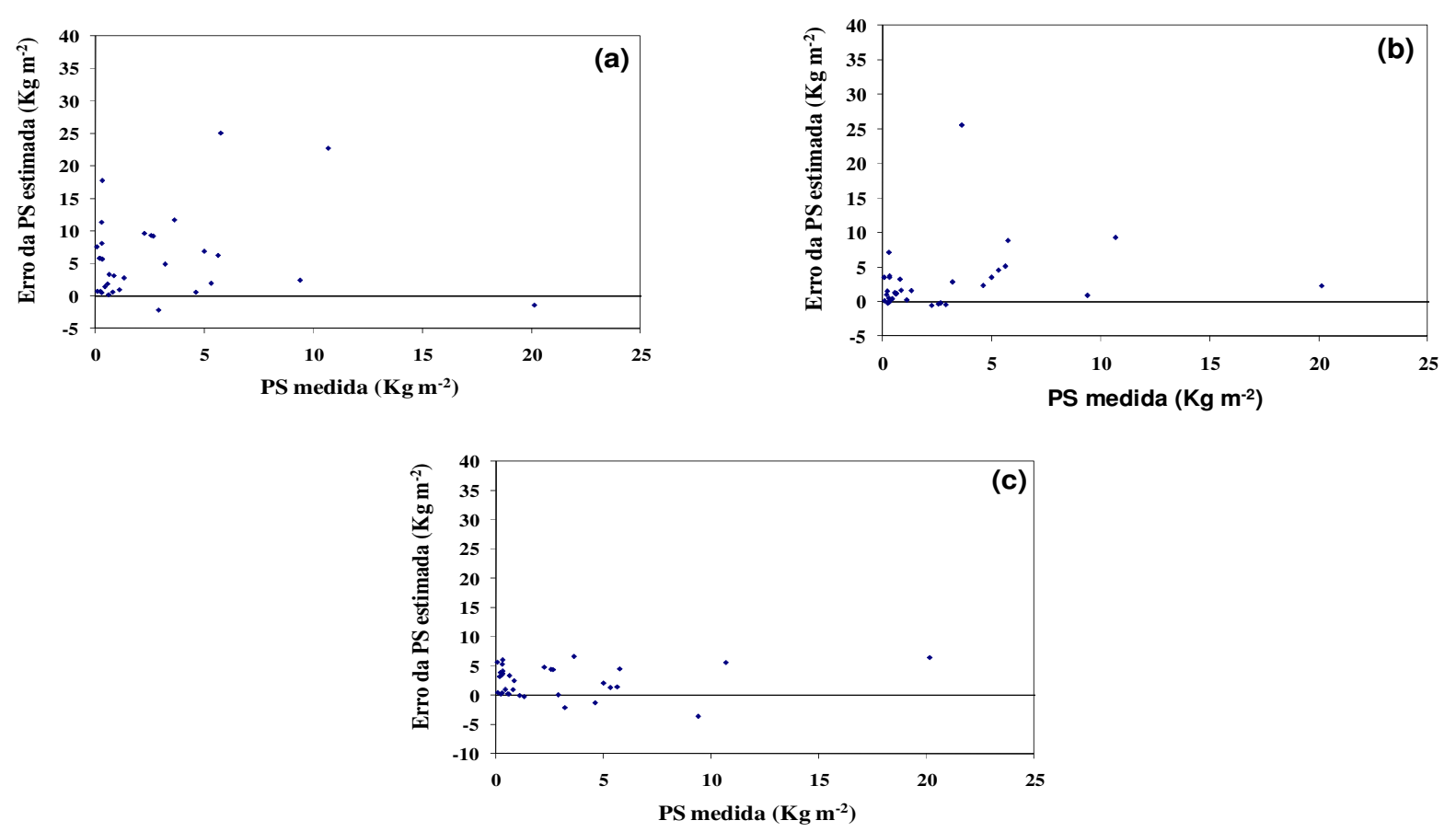

FIGURA 1. Erro das estimativas de perdas de solo (PS) pelos modelos USLE (a), RUSLE (b) e WEPP (c) versus as PS, observadas nas condições de uso e manejo do solo estudadas. Error soil loss (PS) predicted by USLE models (a), RUSLE (b) and WEPP (c) versus the PS observed in the conditions of use and soil management studied.

Na Figura 2, estão apresentadas as perdas de solo médias anuais obtidas experimentalmente versus as perdas estimadas, utilizando-se dos modelos USLE, RUSLE e WEPP para as diferentes condições de uso e manejo do solo das nove localidades estudadas, com suas respectivas linhas de tendência e equações de ajuste. É possível verificar, nessa figura, pelas linhas de tendências apresentadas, que não existem diferenças expressivas entre as estimativas obtidas, utilizando-se dos modelos RUSLE e WEPP para as condições em que ocorrem menores perdas de solo experimentalmente.

No entanto, para elevados valores de perdas de solo, observa-se que as estimativas são bastante diferentes, verificando-se que a linha de tendência ajustada para os dados do modelo WEPP foi a que menos se distanciou da linha de ajuste perfeito, apresentando, desta forma, uma tendência de paralelismo à linha de ajuste perfeito, uma vez que apresenta um coeficiente angular da reta de tendência próximo da unidade $(1,081)$.

Já para os modelos USLE e RUSLE, os coeficientes angulares afastam-se mais da unidade (1,146 e 1,226, respectivamente). Isto indica, aparentemente, que um ajuste nos parâmetros do modelo, tais como erodibilidade (entressulcos e no sulco), tensão crítica de cisalhamento e condutividade hidráulica efetiva, embora esteja fora do âmbito deste estudo, poderia melhorar expressivamente as estimativas pelo modelo WEPP. 


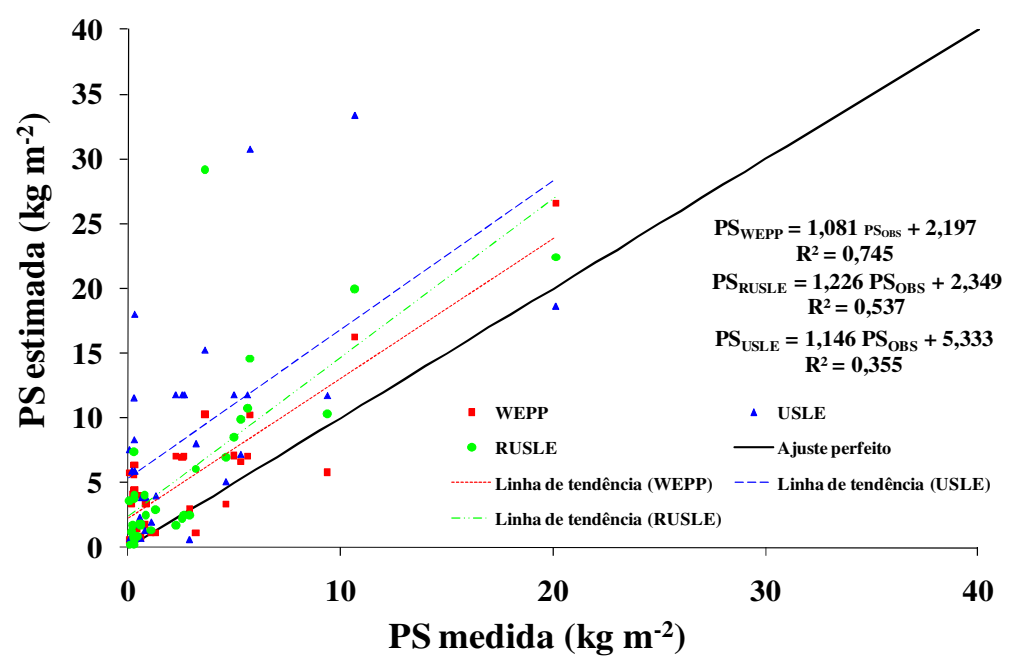

FIGURA 2. Comparação dos valores médios anuais de perdas de solo (PS) observadas e estimadas pelos modelos USLE, RUSLE e WEPP para as condições de uso e manejo do solo estudadas. Average annual soil loss (PS) comparison of measured and predicted values from models USLE, RUSLE and WEPP in the studied conditions of use and soil management of the studied soil.

\section{CONCLUSÕES}

Os três modelos avaliados (USLE, RUSLE e WEPP), para as nove localidades analisadas, superestimam as perdas de solo, independentemente da faixa dos valores de perdas de solo observadas experimentalmente.

Nenhum dos modelos avaliados apresentou a melhor estimativa para todos os cenários de uso e manejo do solo analisados.

O WEPP foi o mais eficiente nas estimativas das perdas de solo, apresentando estimativas mais precisas em $46 \%$ das condições simuladas, seguido pela RUSLE com $42 \%$ e USLE com $12 \%$.

Numa avaliação global, baseada nos indicadores estatísticos estudados, o WEPP apresentou melhor desempenho quando comparado aos modelos USLE e RUSLE.

Embora os três modelos (USLE, RUSLE e WEPP) tenham estimado valores de perdas de solo diferentes, estatisticamente, dos valores observados experimentalmente, o WEPP demonstra ter maior potencial para fazer previsão da erosão para condições edafoclimáticas brasileiras em função do seu melhor desempenho, do coeficiente angular mais próximo da unidade na comparação entre as perdas de solo médias anuais obtidas experimentalmente, versus as perdas estimadas e de ser embasado em processos físicos.

\section{REFERÊNCIAS}

AKSOY, H.; KAVVAS, M. L. A review of hillslope and watershed scale erosion and sediment transport models. Catena, Amsterdan v.64, n.1, p.247-271, 2005.

AMORIM, R.S.S. Avaliação dos modelos de predição da erosão hídrica USLE, RUSLE e WEPP para condições edafoclimáticas brasileiras. 2003. 123 f. Tese (Doutorado em Engenharia Agrícola) - Universidade Federal de Viçosa, Viçosa - MG, 2003.

AVANZI, J.C.; SILVA, M.L.N.; CURI, N.; MELLO, C.R.; FONSECA, S. Calibração e aplicação do modelo MUSLE em uma microbacia hidrográfica nos Tabuleiros Costeiros brasileiros. Revista Brasileira de Engenharia Agrícola e Ambiental, Campina Grande, v.12, n.6, p.563-569, 2008. 
BEZERRA, S.A.; CANTALICE, J.R.B. Erosão entre sulcos em diferentes condições de cobertura do solo, sob cultivo da cana-de-açúcar. Revista Brasileira de Ciência do Solo, Viçosa-MG, v.30, n.3, p.565-573, 2006.

BIUDES, M.S.; VALENTINI, C.M.A.; CAMPELO JÚNIOR, J.H.C.; NOGUEIRA, J.S. Estimativa da evapotranspiração numa pastagem mista, em condições de cerrado, pelos métodos de razão de Bowen e Penman-Monteith. Ciência e Natura, Santa Maria, v.30, n.1, p.71-86, 2008.

CANTALICE, J.R.B.; CASSOL, E.A.; REICHERT, J.M.; BORGES, A.L.O. Hidráulica do escoamento e transporte de sedimentos em sulcos em solo franco argilo arenoso. Revista Brasileira de Ciência do Solo, Viçosa-MG, v.29, n.4, p.597-607, 2005.

CARVALHO FILHO, A.; CENTURION, J.F.; SILVA, R.P.; FURLANI, C.E.A.; CARVALHO, L.C.C. Métodos de preparo do solo: alterações na rugosidade do solo. Engenharia Agrícola, Jaboticabal, v.27, n.1, p.229-237. 2007.

CECÍLIO, R.A.; RODRIGUEZ, R.G.; BAENA, L.G.N.; OLIVEIRA, F.G.; PRUSKI, F.F. Aplicação dos modelos RUSLE e WEPP para a estimativa da erosão hídrica em microbacia hidrográfica de Viçosa (MG). Revista Verde de Agroecologia e Desenvolvimento Sustentável, Mossoró, v.4, n.2, p.39-45, 2009.

COGO, N.P. Uma contribuição à metodologia de estudo das perdas por erosão em condições de chuva natural: I - Sugestões gerais, medição do volume, amostragem e quantificação de solo e água da enxurrada ( $1^{a}$ Aproximação). In: ENCONTRO NACIONAL DE CONSERVAÇÃO DO SOLO, 2., 1978, Passo Fundo. Anais... Passo Fundo: Embrapa-CNPT, 1978a. p.75-97.

DE JONG VAN LIER, Q.; SPAROVEK, G.; FLANAGAN, D.C.; BLOEM, E.M.; SCHNUG, E. Runoff mapping using WEPP erosion model and GIS tools. Computers and Geosciences, New York, v.31, n.10, p.1.270-1.276, 2005.

LEITE, M.H.S. Avaliação das perdas de solo, água e nutrientes em diferentes sistemas de preparo num Latossolo Vermelho-Amarelo sob chuva natural. 2007. 83 f. Dissertação (Mestrado em Agricultura Tropical) - Universidade Federal de Mato Grosso, Cuiabá, 2007.

LEITE, M.H.S.; COUTO, E.G.; AMORIM, S.S.A.; COSTA, E.L.; MARASCHIN, L. Perdas de solo e nutrientes num Latossolo Vermelho-Amarelo Ácrico Típico, com diferentes sistemas de preparo e sob chuva natural. Revista Brasileira de Ciência do Solo, Viçosa-MG, v.21, n.3, p.689699, 2009.

MARTINS FILHO, M.V.; LICCIOTI, T.T.; PEREIRA, G.T.; MARQUES JÚNIOR, J.; SANCHEZ, R.B. Perdas de solo e nutrientes por erosão num Argissolo com resíduos vegetais de cana-deaçúcar. Engenharia Agrícola, Jaboticabal, v.29, n.1, p.8-18, 2009.

NEARING, M.A. Evaluating soil erosion models using measured plot data: accounting for variability in the data. Earth Surface Processes and Landforms, Sussex, v.25, n.9, p.1.035-1.043, 2000.

NUNES, M.C.M.; CASSOL, E.A. Estimativa da erodibilidade em entressulcos de Latossolos do Rio Grande do Sul. Revista Brasileira de Ciência do Solo, Viçosa-MG, v.32, p.2.839-2.845, out/nov. 2008.

PIERI, L.; BITTELLI, M.; WU, J. Q.; DUN, S.; FLANAGAN, D.C.; PISA, P.R.; VENTURA, F.; SALVATORELLI, F. Using the Water Erosion Prediction Project (WEPP) model to simulate fieldobserved runoff and erosion in the Apennines mountain range, Italy. Journal of Hydrology, Amsterdan, v.336, n.1-2, p.84-97, 2007.

RENARD, K.G.; FOSTER, G.R.; WEESIES, G.A.; McCOOL, D.K.; YODER, D.C. Predicting soil erosion by water: a guide to conservation planning with the Revised Universal Soil Loss Equation (RUSLE).Washington: USDA, 1997. 404 p. (Agriculture Handbook, 703). 
RISSE, L.M.; NEARING, M.A.; NICKS, A.D.; LAFLEN, J.M. Assessment of error in the universal soil loss equation. Soil Science Society of America Journal, Madison, v.57, n.3, p.825-833, 1993.

SERGIO, J.; COSTA, C.A.G.; TEIXEIRA, A.S.; ORTEGA, E. Aplicação da USLE e SIG na caracterização de três microbacias hidrográficas no Brasil. Revista Acadêmica: Ciências Agrárias e Ambientais, São José dos Pinhais, v.6, n.2, p.213-221, 2008.

SILVA, A.M.; MELLO, C.R.; CURI, N.; OLIVEIRA, P.M. Simulação da variabilidade espacial da erosão hídrica em uma sub-bacia hidrográfica de Latossolos no sul de Minas Gerais. Revista Brasileira de Ciência do Solo, Viçosa-MG, v.32, n.5, p.2.125-2.134, 2008.

SILVA, D.D.; PRUSKI, F.F.; SCHAEFER, C.E.G.R.; AMORIM, R.S.S.; PAIVA, K.W.N. Efeito da cobertura nas perdas de solo em um Argissolo Vermelho-Amarelo utilizando simulador de chuva. Engenharia Agrícola, Jaboticabal, v.25, n.2, p.409-419, 2005.

TIWARI, A.K.; RISSE, L.M.; NEARING, M.A. Evaluation of WEPP and its comparison with USLE and RUSLE. Transactions of the American Society of Agricultural Engineers, St.Joseph, v.5, n.43, p.1.129-1.135, 2000.

WISCHMEIER, W.H.; SMITH, D.D. Predicting rainfall erosin losses: a guide to conservation planning. Washington: USDA, 1978. 58 p. (Handbook, 537).

ZHANG, X.C.; NEARING, M.A.; RISSE, L.M.; McGREGOR, K.C. Evaluation of runoff and soil loss predictions using natural runoff plot data. Transactions of the American Society of Agricultural Engineers, St Joseph, v.3, n.39, p.855-863, 1996. 